\title{
A New Accurate Neural Network Quantitative Structure- Property Relationship for Prediction of $\theta$ (Lower Critical Solution Temperature) of Polymer Solutions
}

\section{Farhad Gharagheizi *}

Department of Chemical Engineering, Faculty of Engineering, University of Tehran; Fax: (+9821)66957784; Email: fghara@engmail.ut.ac.ir

(Received: 27 June, 2007; published: 5 October, 2007)

\begin{abstract}
In this study, a new neural network quantitative structure-property relationship model for prediction of $\theta(L C S T)$ of polymer solutions is presented. The parameters of this model are eight molecular descriptors which are calculated only from the chemical structure of polymer and solvent. These eight molecular descriptors were selected from 3328 molecular descriptors of polymer and solvent available in polymer solution by genetic algorithm-based multivariate linear regression (GA-MLR) technique. The obtained neural network model can predict the $\theta(L C S T)$ of 169 polymer solutions with mean relative error of $1.67 \%$ and squared correlation coefficient of 0.9736 .
\end{abstract}

\section{Introduction}

Knowledge of phase behavior of polymer solutions is a necessity for industrial and laboratory processes and understanding of it allows for the optimization of design and processing conditions. Partially miscible polymer solutions usually have two boundaries, the upper critical solution temperature (UCST) and lower critical solution temperature (LCST). These two characteristics of partially miscible polymer solutions depend on the molar mass of polymer and also the pressure.

The theta temperature is defined as the critical miscibility temperature at the limit of infinite molar mass. Since a solution may exhibit two critical miscibility temperatures, a polymer solution may display two theta temperatures.

In the case of exothermic solutions, an increasing of temperature leads to less positive second virial coefficients and the theta temperature corresponds to a LCST in the limit of infinite molar mass $(\theta(L C S T))$. The theta temperature corresponds to an UCST in the case of endothermic solutions ( $\theta(U C S T))$ [1].

$\theta(L C S T)$ is the LCST at infinite molar mass, as a result, it is not affected by molar mass of polymers. Also, it can serve as an upper temperature limit for polymer processing in the form of solution. Solvent systems that exhibit this LCST behavior have been suggested for applications where partial miscibility above and below LCST offers advantages [2, 3].

Prediction of physicochemical properties of materials from their molecular structure has been one of the wishes of the scientists and engineers for a long time. One of the useful methods which applied for this purpose is quantitative structure-property 
relationship (QSPR). QSPR is defined as a mathematical model which predicts the physical, mechanical, or chemical properties of materials from their chemical structures. The main goal of QSPR studies is to find a relationship between the structure of a compound expressed in terms of numeric characteristics associated with its chemical structure (which are called molecular descriptors) and the properties of interest.

Once a correlation between structure and desired property is found, any number of compounds, including those not yet synthesized, can be readily screened on computer in order to select structures with the properties desired. Thus QSPR approach conserves resources and accelerates the process of development of new molecules for use in any purpose. Also, since theoretical descriptors derived solely from the molecular structure would be involved, the relation should, in principle, be applicable to any chemical structure.

There are more than thousand of molecular descriptors available in the literature to represent the molecular structure, and one usually faces the problem of selecting those which are the most representative for the property under consideration [4].

There are many methods for estimating LCSTs, including $\theta(L C S T)$. As stated by Melagraki et al. [5], these methods can be divided into three main groups. The first group is those methods that have a solid theoretical background but they require vapor-liquid equilibrium, and also liquid-liquid equilibrium experimental data for obtaining their parameters [6-8]. The ability of these methods for prediction of new systems is unknown. The second group of methods for estimating $\theta(L C S T)$ is the empirical models which relates $\theta(L C S T)$ to other physicochemical properties [9-12, 13]. These correlations are simple and have reasonable accuracy, but they cannot be used to systems for which the required physicochemical properties are not available. The third group of methods for estimating $\theta(L C S T)$ is QSPR analysis. These types of methods have two main advantages in comparison to previous methods. For development of a QSPR model, molecular descriptors which, are computed only from chemical structure of compounds, are applied. As a result, these types of methods do not need any additional information (i.e. other physical properties or vapor-liquid or liquid-liquid equilibrium data). On the other hand, the prediction power of these types of models are always checked during development of the models, as a result, these types of models can be accurately applied for any regular systems.

The first attempt to apply QSPR analysis for prediction of $\theta(L C S T)$ is related to the work of Liu and Zhong [14] who applied the molecular connectivity indices (a special group of molecular descriptors [4]) for prediction of $\theta(L C S T)$ for polymer solutions with $R^{2}=0.77$ and mean relative error (MRE) of 5.44\%. It is noted that Liu and Zhong [14] could not succeed to develop a unified model for prediction of $\theta(L C S T)$ for polymer solutions. They used eight equations for prediction of $\theta(L C S T)$ for solutions of eight polymers.

First attempt to develop a unified model for prediction of $\theta(L C S T)$ for polymer solutions by QSPR analysis, is related to the recent work of Melagraki et al [5] who obtained a nine-parameter equation for prediction of $\theta(L C S T)$ for polymer solutions by means of multivariate linear regression (MLR) technique, with $R^{2}=0.8660$ and MRE of $3.73 \%$. 
Also Xu et al. [15] recently tried to develop a QSPR model for prediction of $\theta($ LCST ) for polymer solutions by means of MLR technique, based on topological descriptors (a special group of molecular descriptors [4]). Their ten-parameter equation predicts $\theta(L C S T)$ for polymer solutions with $R^{2}=0.8874$ and MRE of $3.49 \%$.

There are several comments about the previous QSPR studies of Liu and Zhong [13], Melagraki et al [5], and Xu et al. [15]. In previous works, all authors tried to develop their QSPR models based on a special group of molecular descriptors (connectivity indices in work of Liu and Zhong [14], 3D descriptors in the work of Melagraki et al [5], and topological descriptors in the work of $\mathrm{Xu}$ et al. [15]). This phenomenon caused the obtained results to be not the best results which can be obtained by QSPR analysis. Another comment is related to the method of selection of most statistically effective molecular descriptors on $\theta(L C S T)$ of polymer solutions. All three groups of authors $[5,14,15]$ have used the MLR technique. This technique has several disadvantages that are presented elsewhere [16]. Another important comment is related to the study of the nonlinear behavior of the molecular descriptors on $\theta(L C S T)$ of polymer solutions, as a complementary work (such as application of neural networks).

In the present study, first the genetic algorithm based-multivariate linear regression (GA-MLR) is applied to select the most statistically effective molecular descriptors on the $\theta(L C S T)$ of polymer solutions from a pool of 3328 molecular descriptors which belong to many types of molecular descriptors.

Then by means of the results of GA-MLR, a feed forward neural network is constructed for predicting $\theta(L C S T)$ of polymer solutions.

\section{Results and Discussion}

In this section, it is better we refer to comments which were presented in introduction part of this paper. First comment is related to the types of molecular descriptors which should be used to develop a QSPR model. Physical properties are usually the result of many types of interactions, as a result, in this work 3228 molecular descriptors from many types, were used. Diversity of molecular descriptors can represent many interactions between molecules. Also, the GA-MLR was applied to find the most statistically effective molecular descriptors of polymer and solvent on $\theta(L C S T)$. Comparison between the presented results of Tab. 5 and previous works $[5,14]$ shows that the presented multi-linear equation (see Tab. 3), is quite as accurate as previous works, but there are eight parameters. The obtained small modification in first part of calculations is the result of application different types of molecular descriptors and application of a powerful method for selection of molecular descriptors from the pool of 3328 molecular descriptors (GA-MLR). It should be noted that Melagraki et al. [5] presented a nine-parameter multi-linear equation, and Xu et al. [15] presented a ten-parameter multi-linear equation for prediction of $\theta(L C S T)$ with the same data set.

Nine-parameter multi-linear equation proposed by Melagraki et al. [5] shows MRE of $3.76 \%$ over experimental data set (both training and test sets) which was used in this study. Also the ten-parameter multi-linear equation proposed by $\mathrm{Xu}$ et al. [15] shows MRE of $4.95 \%$ over this data set. The eight-parameter multi-linear model presented in this study shows MRE of $4.04 \%$. This model is better than the model proposed by $\mathrm{Xu}$ et al. [15] and has two parameters less than that model. But, it is not possible to 
compare this model with the model proposed by Melagraki et al. [5], because, MRE of the model proposed by Melagraki et al. [5] has better than MRE of this study, but has one parameter more than this model. As a result, application of diverse 3328 molecular descriptors and Experimental $\theta$ (LCST) also application of GA-MLR caused small modification in comparison to previous models.

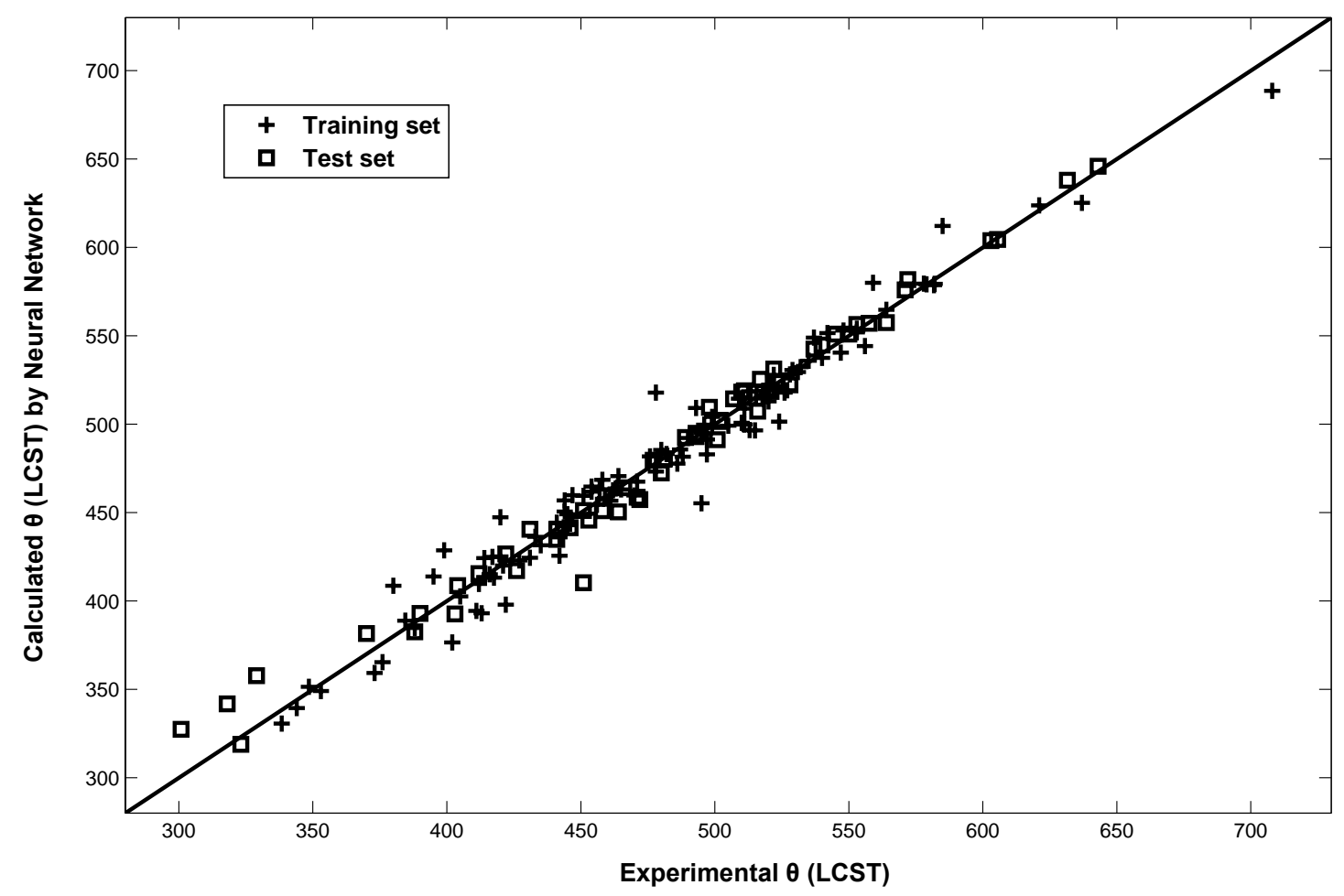

Fig. 1. Comparison between the best obtained FFNN results and the experimental.

Tab. 1. The statistical parameters of the best obtained FFNN.

\begin{tabular}{ll}
\hline Statistical Parameter & Value \\
\hline $\begin{array}{l}\text { Standard Multiple Correlation Coefficient for Training set } \\
\left(\mathrm{R}_{\text {Train }}{ }^{2}\right)\end{array}$ & 0.978 \\
Standard Multiple Correlation Coefficient for Test set $\left(\mathrm{R}_{\text {Test }}{ }^{2}\right)$ & 0.9722 \\
Standard Multiple Correlation Coefficient for Training set + & \\
Test set ( $\left.\mathrm{R}^{2}\right)$ & 0.9736 \\
Mean Relative Error for Training Set (MRE & $1.68 \%$ \\
Mean Relative Error for Test Set (MRE & $1.66 \%$ \\
Mean Relative Error for Training set + Test Set (MRE) & $1.67 \%$ \\
\hline
\end{tabular}

The second comment which is presented in introduction part of this paper is related to the nonlinear study for QSPR analysis. The major modification in the presented work is related to the application of neural networks for nonlinear study of the obtained multi-linear equation by GA-MLR. 
Application of FFNN considerably modifies the results of the GA-MLR. This considerable modification was presented in Tab. 1. As can be found from Tab. 1, the obtained FFNN shows MRE of $1.67 \%$ over data set which shows considerable modification in comparison with the previous model.

The predicted values of $\theta(L C S T)$ by GA-MLR, and FFNN in comparison to the experimental data are presented in Tab. 6. Also, the systems which were used as training set and test set are shown.

Tab. 2. Comparison between GA-MLR and FFNN results and experimental data.

\begin{tabular}{|c|c|c|c|c|c|c|c|}
\hline \multirow{2}{*}{ ID } & \multirow{2}{*}{\multicolumn{2}{|c|}{ Polymer Solvent }} & \multirow{2}{*}{ Experimental } & \multicolumn{2}{|c|}{ GA-MLR } & \multicolumn{2}{|c|}{ FFNN } \\
\hline & & & & Predicted & status & Predicted & Status \\
\hline 1 & P4MP1 & n-Butane & 388 & 408.07 & Training & 382.57 & Test \\
\hline 2 & P4MP1 & n-Pentane & 441 & 444.43 & Training & 434.82 & Test \\
\hline 3 & P4MP1 & n-Heptane & 522 & 519.56 & Training & 527.84 & Training \\
\hline 4 & P4MP1 & n-Octane & 553 & 541.91 & Training & 554.02 & Training \\
\hline 5 & P4MP1 & 2-Methyl butane & 431 & 431.76 & Training & 424.42 & Training \\
\hline 6 & P4MP1 & 2,2-Dimethyl butane & 462 & 461.94 & Training & 462.58 & Training \\
\hline 7 & P4MP1 & 2,2-Dimethyl pentane & 499 & 507.14 & Training & 504.09 & Training \\
\hline 8 & P4MP1 & 2,2,3-Trimethyl butane & 521 & 501.97 & Training & 518.46 & Test \\
\hline 9 & P4MP1 & Cyclopentane & 505 & 516.15 & Training & 499.13 & Training \\
\hline 10 & P4MP1 & n-Hexane & 487 & 480.60 & Test & 485.69 & Training \\
\hline 11 & P4MP1 & n-Nonane & 579 & 567.67 & Test & 579.13 & Training \\
\hline 12 & P4MP1 & 2,4-Dimethyl pentane & 499 & 495.06 & Test & 498.94 & Training \\
\hline 13 & P4MP1 & 3-Ethyl pentane & 532 & 505.57 & Test & 532.20 & Training \\
\hline 14 & PB1 & n-Pentane & 421 & 447.86 & Training & 420.00 & Training \\
\hline 15 & PB1 & n-Heptane & 509 & 522.55 & Training & 514.42 & Training \\
\hline 16 & PB1 & n-Octane & 540 & 544.94 & Training & 537.53 & Training \\
\hline 17 & PB1 & 2-Methyl butane & 416 & 435.14 & Training & 415.05 & Training \\
\hline 18 & PB1 & 2,2-Dimethyl butane & 444 & 465.25 & Training & 456.86 & Training \\
\hline 19 & PB1 & 2,2,3-Trimethyl butane & 507 & 504.91 & Training & 514.33 & Test \\
\hline 20 & PB1 & 2,5-Dimethyl hexane & 519 & 514.09 & Training & 515.98 & Training \\
\hline 21 & PB1 & 3-Ethyl pentane & 523 & 507.61 & Training & 519.43 & Training \\
\hline 22 & PB1 & 3,4-Dimethyl hexane & 559 & 555.70 & Training & 579.99 & Training \\
\hline 23 & PB1 & n-Hexane & 464 & 483.12 & Test & 470.59 & Training \\
\hline 24 & PB1 & n-Nonane & 564 & 570.19 & Test & 557.42 & Test \\
\hline 25 & PB1 & 2,4-Dimethyl pentane & 480 & 497.59 & Test & 485.39 & Training \\
\hline 26 & PB1 & 2,3-Dimethyl pentane & 517 & 516.23 & Test & 525.38 & Test \\
\hline 27 & PB1 & Cyclopentane & 498 & 518.14 & Test & 509.51 & Test \\
\hline 28 & PBD & n-Hexane & 373 & 410.71 & Training & 359.29 & Training \\
\hline 29 & PBD & 2,2,3-Trimethyl butane & 414 & 431.25 & Training & 413.50 & Training \\
\hline 30 & PBD & n-Octane & 390 & 475.49 & Training & 392.96 & Test \\
\hline 31 & PBD & Ethyl propyl ketone & 510 & 449.12 & Training & 500.79 & Training \\
\hline
\end{tabular}




\begin{tabular}{|c|c|c|c|c|c|c|c|}
\hline 32 & PBD & Diethyl ketone & 481 & 492.91 & Training & 480.21 & Test \\
\hline 33 & PBD & 2-Methyl hexane & 370 & 431.33 & Test & 381.55 & Test \\
\hline 34 & PBD & 2,2,4-Trimethyl pentane & 388 & 448.32 & Test & 384.77 & Training \\
\hline 35 & PBD & Propylene oxide & 414 & 435.54 & Test & 424.19 & Training \\
\hline 36 & PDMS & n-Pentane & 453 & 450.86 & Training & 445.67 & Test \\
\hline 37 & PDMS & n-Heptane & 528 & 526.30 & Training & 528.18 & Training \\
\hline 38 & PDMS & n-Octane & 553 & 548.48 & Training & 551.90 & Training \\
\hline 39 & PDMS & n-Dodecane & 643 & 658.30 & Training & 645.91 & Test \\
\hline 40 & PDMS & n-Cetane & 708 & 719.82 & Training & 688.53 & Training \\
\hline 41 & PDMS & n-Hexane & 493 & 487.55 & Test & 492.96 & Test \\
\hline 42 & PDMS & n-Decane & 603 & 604.85 & Test & 603.83 & Test \\
\hline 43 & $\mathrm{PE}$ & n-Hexane & 411 & 433.08 & Training & 394.37 & Training \\
\hline 44 & $\mathrm{PE}$ & n-Heptane & 459 & 471.70 & Training & 457.89 & Training \\
\hline 45 & $\mathrm{PE}$ & n-Nonane & 531 & 519.01 & Training & 529.45 & Training \\
\hline 46 & $\mathrm{PE}$ & n-Decane & 557.55 & 549.24 & Training & 557.06 & Test \\
\hline 47 & $\mathrm{PE}$ & n-Dodecane & 605.55 & 599.62 & Training & 604.47 & Test \\
\hline 48 & $\mathrm{PE}$ & $\mathrm{n}$-Tridecane & 631.55 & 610.28 & Training & 637.99 & Test \\
\hline 49 & $\mathrm{PE}$ & 2,2-Dimethyl pentane & 399 & 460.26 & Training & 428.65 & Training \\
\hline 50 & PE & 2,2,3-Trimethyl butane & 444 & 454.50 & Training & 450.53 & Training \\
\hline 51 & $\mathrm{PE}$ & 2,3-Dimethyl pentane & 463 & 465.54 & Training & 463.66 & Training \\
\hline 52 & $\mathrm{PE}$ & 3-Ethyl pentane & 471 & 456.90 & Training & 458.65 & Test \\
\hline 53 & $\mathrm{PE}$ & 2,2,4,4-Tetramethyl pentane & 513 & 498.06 & Training & 496.65 & Training \\
\hline 54 & $\mathrm{PE}$ & 2,3,4-Trimethyl hexane & 545 & 552.04 & Training & 550.99 & Test \\
\hline 55 & $\mathrm{PE}$ & Methyl cyclopentane & 488 & 488.24 & Training & 481.58 & Training \\
\hline 56 & $\mathrm{PE}$ & Methyl cyclohexane & 537 & 530.54 & Training & 548.90 & Training \\
\hline 57 & $\mathrm{PE}$ & 1-Octanol & 621 & 549.92 & Training & 623.79 & Training \\
\hline 58 & $\mathrm{PE}$ & n-Pentyl acetate & 528 & 565.71 & Training & 522.22 & Test \\
\hline 59 & $\mathrm{PE}$ & n-Pentane & 353 & 396.07 & Test & 349.03 & Training \\
\hline 60 & $\mathrm{PE}$ & n-Octane & 496 & 493.99 & Test & 499.66 & Training \\
\hline 61 & PE & $\mathrm{n}$-Undecane & 581.75 & 577.99 & Test & 578.66 & Training \\
\hline 62 & $\mathrm{PE}$ & 2,4-Dimethyl pentane & 395 & 446.83 & Test & 413.85 & Training \\
\hline 63 & $\mathrm{PE}$ & 2,2,4-Trimethyl pentane & 495 & 472.27 & Test & 455.28 & Training \\
\hline 64 & $\mathrm{PE}$ & 3,4-Dimethyl hexane & 515 & 505.07 & Test & 496.46 & Training \\
\hline 65 & $\mathrm{PE}$ & Cyclopentane & 472 & 467.38 & Test & 457.33 & Test \\
\hline 66 & $\mathrm{PE}$ & Cyclohexane & 518 & 513.89 & Test & 518.12 & Test \\
\hline 67 & $\mathrm{PE}$ & n-Butyl acetate & 490 & 540.88 & Test & 492.14 & Training \\
\hline 68 & PIB & Cyclopentane & 461 & 447.28 & Training & 456.64 & Training \\
\hline 69 & PIB & Cyclohexane & 516 & 492.66 & Training & 507.38 & Test \\
\hline 70 & PIB & Cycloheptane & 572 & 615.63 & Training & 581.80 & Test \\
\hline 71 & PIB & Cyclooctane & 637 & 665.59 & Training & 625.15 & Training \\
\hline 72 & PIB & n-Heptane & 442 & 452.47 & Training & 436.00 & Training \\
\hline 73 & PIB & n-Octane & 477 & 474.57 & Training & 479.53 & Test \\
\hline 74 & PIB & n-Dodecane & 582 & 580.77 & Training & 579.41 & Training \\
\hline 75 & PIB & 2-Methyl butane & 318 & 368.13 & Training & 341.82 & Test \\
\hline
\end{tabular}




\begin{tabular}{|c|c|c|c|c|c|c|c|}
\hline 76 & PIB & 2-Methyl pentane & 376 & 404.67 & Training & 365.31 & Training \\
\hline 77 & PIB & 3-Methyl pentane & 405 & 401.90 & Training & 402.58 & Training \\
\hline 78 & $\mathrm{PIB}$ & 3-Methyl hexane & 446 & 441.15 & Training & 441.44 & Test \\
\hline 79 & PIB & 3-Ethyl pentane & 458 & 436.98 & Training & 451.08 & Test \\
\hline 80 & PIB & 2,3-Dimethyl pentane & 451 & 445.91 & Training & 459.44 & Training \\
\hline 81 & PIB & 2,4-Dimethyl pentane & 403 & 428.64 & Training & 392.61 & Test \\
\hline 82 & PIB & 2,2,3-Trimethyl butane & 445 & 434.41 & Training & 449.04 & Training \\
\hline 83 & PIB & Ethyl cyclopentane & 524 & 484.19 & Training & 501.43 & Training \\
\hline 84 & PIB & 3-Methyl heptane & 478 & 470.73 & Training & 473.18 & Training \\
\hline 85 & PIB & 2,2-Dimethyl hexane & 454 & 464.89 & Training & 461.86 & Training \\
\hline 86 & PIB & 2,5-Dimethyl hexane & 446 & 444.12 & Training & 443.70 & Training \\
\hline 87 & PIB & 3.4-Dimethyl hexane & 497 & 485.20 & Training & 491.30 & Training \\
\hline 88 & PIB & n-Propyl cyclopentane & 547 & 544.48 & Training & 540.41 & Training \\
\hline 89 & $\mathrm{PIB}$ & n-Pentane & 344 & 376.74 & Test & 339.35 & Training \\
\hline 90 & PIB & n-Hexane & 402 & 413.03 & Test & 376.56 & Training \\
\hline 91 & $\mathrm{PIB}$ & Methyl cyclopentane & 478 & 511.77 & Test & 517.83 & Training \\
\hline 92 & PIB & 2-Methyl hexane & 426 & 435.94 & Test & 417.20 & Test \\
\hline 93 & PIB & 2,2-Dimethyl pentane & 404 & 439.37 & Test & 408.68 & Test \\
\hline 94 & PIB & 3,3-Dimethyl pentane & 451 & 434.29 & Test & 451.11 & Test \\
\hline 95 & PIB & Methyl cyclohexane & 526 & 511.77 & Test & 517.83 & Training \\
\hline 96 & PIB & 2-Methyl heptane & 466 & 462.83 & Test & 464.06 & Test \\
\hline 97 & PIB & 2,4-Dimethyl hexane & 458 & 465.74 & Test & 468.47 & Training \\
\hline 98 & PIB & 2,2,4-Trimethyl pentane & 435 & 452.94 & Test & 431.51 & Training \\
\hline 99 & PIB & n-Decane & 535 & 530.33 & Test & 535.83 & Training \\
\hline 100 & PMMA & Methyl acetate & 441 & 453.85 & Training & 444.24 & Training \\
\hline 101 & PMMA & Ethyl acetate & 478 & 468.89 & Training & 476.46 & Training \\
\hline 102 & PMMA & Ethyl propyl ketone & 511 & 552.28 & Training & 508.76 & Training \\
\hline 103 & PMMA & Diethyl ketone & 497 & 520.43 & Training & 482.87 & Training \\
\hline 104 & PMMA & Butyl chloride & 454 & 444.56 & Test & 464.73 & Training \\
\hline 105 & PMMA & Dipropyl ketone & 522 & 571.84 & Test & 531.18 & Test \\
\hline 106 & PMMA & Methyl ethyl ketone & 464 & 447.32 & Test & 450.42 & Test \\
\hline 107 & PMMA & Tetrahydrofuran & 519.5 & 505.83 & Test & 516.06 & Training \\
\hline 108 & PMS & Cyclopentane & 417.6 & 385.57 & Training & 413.19 & Training \\
\hline 109 & PMS & Cyclohexane & 456 & 432.97 & Training & 454.38 & Training \\
\hline 110 & PMS & Butyl chloride & 412 & 356.99 & Training & 409.62 & Training \\
\hline 111 & PMS & n-Butyl acetate & 446.9 & 463.43 & Training & 459.85 & Training \\
\hline 112 & PMS & n-Hexyl acetate & 500.9 & 521.33 & Training & 491.23 & Test \\
\hline 113 & PMS & Methyl cyclohexane & 431 & 452.23 & Test & 440.50 & Test \\
\hline 114 & PMS & n-Pentyl acetate & 475.8 & 492.24 & Test & 481.81 & Training \\
\hline 115 & $\mathrm{PP}$ & n-Pentane & 422 & 416.47 & Training & 397.90 & Training \\
\hline 116 & $\mathrm{PP}$ & n-Hexane & 470 & 452.54 & Training & 459.67 & Training \\
\hline 117 & $\mathrm{PP}$ & n-Octane & 542 & 513.88 & Training & 551.52 & Training \\
\hline 118 & PP & n-Nonane & 571 & 538.85 & Training & 575.95 & Test \\
\hline 119 & PP & 2,2-Dimethyl butane & 441 & 434.08 & Training & 440.33 & Test \\
\hline
\end{tabular}




\begin{tabular}{|c|c|c|c|c|c|c|c|}
\hline 120 & PP & 2,3-Dimethyl butane & 465 & 443.20 & Training & 463.07 & Training \\
\hline 121 & $\mathrm{PP}$ & 2,2-Dimethyl pentane & 489 & 479.10 & Training & 492.49 & Test \\
\hline 122 & $\mathrm{PP}$ & 2,2,3-Trimethyl butane & 511 & 474.08 & Training & 499.80 & Training \\
\hline 123 & $\mathrm{PP}$ & 2,3-Dimethyl pentane & 513 & 485.44 & Training & 515.02 & Test \\
\hline 124 & $\mathrm{PP}$ & 3-Ethyl pentane & 520 & 476.87 & Training & 512.87 & Training \\
\hline 125 & PP & 2,2,4,4-Tetramethyl pentane & 548 & 518.35 & Training & 552.98 & Training \\
\hline 126 & $\mathrm{PP}$ & 2,3,4-Trimethyl hexane & 585 & 571.18 & Training & 612.07 & Training \\
\hline 127 & $\mathrm{PP}$ & Methyl cyclopentane & 518 & 508.92 & Training & 518.87 & Training \\
\hline 128 & $\mathrm{PP}$ & Methyl cyclohexane & 564 & 550.52 & Training & 564.71 & Training \\
\hline 129 & $\mathrm{PP}$ & Diethyl ether & 420 & 427.20 & Training & 447.31 & Training \\
\hline 130 & $\mathrm{PP}$ & n-Heptane & 511 & 491.96 & Test & 518.86 & Test \\
\hline 131 & $\mathrm{PP}$ & 2-Methyl butane & 413 & 404.07 & Test & 393.00 & Training \\
\hline 132 & PP & 2,4-Dimethyl pentane & 481 & 467.40 & Test & 480.39 & Test \\
\hline 133 & $\mathrm{PP}$ & 2,2,4-Trimethyl pentane & 510 & 492.84 & Test & 518.10 & Test \\
\hline 134 & $\mathrm{PP}$ & 3,4-Dimethyl hexane & 553 & 525.65 & Test & 556.35 & Test \\
\hline 135 & $\mathrm{PP}$ & Cyclopentane & 495 & 487.96 & Test & 494.93 & Training \\
\hline 136 & $\mathrm{PP}$ & Cyclohexane & 540 & 534.47 & Test & 544.73 & Test \\
\hline 137 & PP1 & n-Pentane & 433 & 450.09 & Training & 436.23 & Training \\
\hline 138 & PP1 & n-Hexane & 482 & 485.83 & Training & 483.10 & Training \\
\hline 139 & PP1 & n-Octane & 556 & 547.04 & Training & 544.13 & Training \\
\hline 140 & PP1 & 2-Methyl butane & 422 & 437.58 & Training & 426.65 & Test \\
\hline 141 & PP1 & 2,4-Dimethyl pentane & 493 & 500.40 & Training & 494.85 & Test \\
\hline 142 & PP1 & 2,2-Dimethyl pentane & 502 & 512.35 & Training & 501.51 & Test \\
\hline 143 & PP1 & 2,3-Dimethyl pentane & 529 & 518.50 & Training & 530.58 & Training \\
\hline 144 & PP1 & 3-Methyl heptane & 537 & 543.89 & Training & 542.46 & Test \\
\hline 145 & PP1 & n-Heptane & 522 & 524.74 & Test & 521.70 & Training \\
\hline 146 & PP1 & 2,2-Dimethyl butane & 457 & 467.06 & Test & 463.15 & Training \\
\hline 147 & PP1 & 2,2,4-Trimethyl pentane & 527 & 525.62 & Test & 519.13 & Training \\
\hline 148 & PP1 & Cyclopentane & 502 & 520.74 & Test & 502.19 & Test \\
\hline 149 & PPCS & Isobutyl acetate & 348.5 & 309.64 & Training & 351.40 & Training \\
\hline 150 & PPCS & Ethyl carbitol & 300.8 & 333.27 & Training & 327.34 & Test \\
\hline 151 & PPCS & n-Butyl carbitol & 323.1 & 345.39 & Training & 318.89 & Test \\
\hline 152 & PPCS & tert-Butyl acetate & 338.4 & 299.83 & Test & 330.60 & Training \\
\hline 153 & PS & Cyclopentane & 427 & 409.70 & Training & 422.88 & Training \\
\hline 154 & PS & Cyclohexane & 486 & 455.17 & Training & 477.71 & Training \\
\hline 155 & PS & Methyl cyclopentane & 417 & 432.93 & Training & 424.89 & Training \\
\hline 156 & PS & Methyl cyclohexane & 480 & 474.08 & Training & 472.37 & Test \\
\hline 157 & PS & Benzene & 523 & 516.27 & Training & 523.56 & Test \\
\hline 158 & PS & Toluene & 550 & 601.57 & Training & 550.97 & Test \\
\hline 159 & PS & Ethyl acetate & 412 & 410.70 & Training & 415.48 & Test \\
\hline 160 & PS & n-Propyl acetate & 451 & 424.34 & Training & 410.29 & Test \\
\hline 161 & PS & i-Propyl acetate & 380 & 421.05 & Training & 408.55 & Training \\
\hline 162 & PS & Ethyl n-butyrate & 471 & 458.21 & Training & 467.45 & Training \\
\hline 163 & PS & Isobutyl acetate & 445 & 457.55 & Training & 442.79 & Training \\
\hline
\end{tabular}




\begin{tabular}{|c|c|c|c|c|c|c|c|}
\hline 164 & PS & sec-Butyl acetate & 442 & 453.39 & Training & 425.55 & Training \\
\hline 165 & PS & tert-Butyl acetate & 329 & 425.93 & Test & 357.76 & Test \\
\hline 166 & PS & Diethyl malonate & 578 & 595.66 & Test & 579.46 & Training \\
\hline 167 & PS & i-Amyl acetate & 493 & 514.49 & Test & 509.17 & Training \\
\hline 168 & PS & Methyl acetate & 384.5 & 394.48 & Test & 388.80 & Training \\
\hline 169 & PS & Methyl ethyl ketone & 420 & 401.97 & Test & 425.14 & Training \\
\hline
\end{tabular}

\section{Conclusions}

In this paper, a complete QSPR analysis was performed for prediction of $\theta(L C S T)$ polymer solutions. This QSPR study contains two parts: linear study and nonlinear study. The result of first part is input of the second part.

The linear part contains selecting most statistically effective molecular descriptors on $\theta$ (LCST) polymer solutions. This part was done by means of GA-MLR technique based on 3328 molecular descriptors of polymer and solvent. The result of this part is an eight-parameter multi-linear equation. All eight parameters of this equation can be calculated only from the chemical structure of polymer and solvent available in a polymer solution. Also, this equation has two parameters less than the obtained equations by previous researchers with the same accuracy.

The nonlinear part of this study is related to generating a neural network on the eight parameters of multi-linear model obtained by GA-MLR in first part of this study. In this part the eight parameters of the multi-linear was used as input of a FFNN and finally, an optimized 8-10-1 FFNN was presented for prediction of $\theta(L C S T)$ polymer solutions.

This optimized FFNN is accurate and can be applied for prediction of $\theta($ LCST ) any regular polymer solutions.

\section{Materials and Methods}

\section{Data set}

The data set which is used in this study contains the 169 experimental $\theta(L C S T)$ for polymer solutions including 12 polymers and 67 solvents. This data set is compiled by Liu and Zhong [17]. This data set was used to develop QSPR models by Liu and Zhong [14], Melagraki et al [5], and Xu et al. [15]. As a result, comparison between this study and their studies is possible.

\section{Determination of Molecular Descriptors}

Each polymer solution is formed from a polymer and a solvent. As a result the phase behavior of polymer solution is a function of the both polymer and solvent and we must consider all molecular descriptors of polymers and also all molecular descriptors of solvents.

In this work, the monomer compounds were used to determine the molecular descriptors of polymers. After providing the data set, all molecules (polymers and solvents) were drawn into Hyperchem software [18] and pre-optimized using the $\mathrm{MM}+$ molecular mechanics force field. A more precise optimization was done with 
AM1 method in Hyperchem and thereafter, molecular descriptors were calculated using the optimized molecular structure of compounds by means of Dragon software [19]. Dragon can calculate 1664 molecular descriptors for any optimized chemical structure. These molecular descriptors contain many types of molecular descriptors such as connectivity indices and topological descriptors. For more information about the types of the molecular descriptors which Dragon can calculate, refer to Dragon software user's guide [19].

In this part, totally 3328 molecular descriptors for each polymer solutions were computed by means of Dragon (1664 molecular descriptors for polymer and 1664 molecular descriptors for solvent).

\section{GA-MLR Calculations}

After calculation of molecular descriptors, we can develop our models for prediction of $\theta(L C S T)$. In QSPR study, the main problem is to find a multivariate linear equation with minimum input parameters (molecular descriptors) which can predict the desired property $(\theta(L C S T))$ with the best accuracy. This problem is classified in the field of subset variable selection. In other words, we should select the best subset variables from the set of 3328 molecular descriptors which can predict $\theta(L C S T)$ with the best accuracy.

One of the best solutions of this problem is genetic algorithm-based multivariate linear regression (GA-MLR). In this method, genetic algorithm is used for selection of best subset variables with respect to an objective function which is usually defined as the sum of square error between obtained values of the model and the corresponding values in dataset. In this work, we use GA-MLR algorithm which has been proposed by Leardi et al. [20] and has been successfully used by the author of this paper in his previous works [15, 21-23]. This algorithm has been designed for feature selection by means of genetic algorithm search which is suitable for this problem.

In the first part of calculations, data set was divided into two sub-datasets. First is training set and contains 112 polymer solutions and, second is test set and contains 57 polymer solutions. Training set is used to develop the model and test set is only used to check the validity of model and is not used to develop model. In this study we used the same training set and test set which had been used by Xu et al. [15].

Inputs of our program are the pool of 3328 molecular descriptors, and the number of desired molecular descriptors which we want to obtain in our study. In the first step, we started with one molecular descriptor models. Based on the training set, the best multivariate one molecular descriptor linear model was obtained, GA-MLR. In next step, we should examine two molecular descriptors multivariate linear models. After obtaining the best two molecular descriptors model by GA-MLR, we should compare the best one molecular descriptor model with the best two molecular descriptors multivariate linear model. In this comparison, we should consider the effect of increase in number of molecular descriptors on the increasing accuracy of these two models. If the increase in the number of molecular descriptors showed a sensible increase in accuracy of model, we must continue this procedure and increase number of molecular descriptors to three and then we should find the best three molecular descriptors multivariate linear model, by GA-MLR. If the increase in the number of molecular descriptors did not show any sensible effect on accuracy of model, we must stop our search. The best model has been obtained. 
As the procedure presented in previous paragraph, we must repeat our work to find the best three, four, five, and so on, molecular descriptors multivariate linear models.

Tab. 3. The best obtained multi-linear equation by GA-MLR and its properties.

\begin{tabular}{|c|c|c|c|c|c|}
\hline ID & Variable & $\begin{array}{l}\text { Regression } \\
\text { Coefficient }\end{array}$ & $\begin{array}{l}\text { Errors } \\
\text { Regression } \\
\text { Coefficient }\end{array}$ & $\begin{array}{l}\text { Confidence } \\
\text { Intervals } \\
(0.95)\end{array}$ & $\begin{array}{l}\text { Standard } \\
\text { Regression } \\
\text { Coefficient }\end{array}$ \\
\hline 0 & $\begin{array}{l}\text { intercept } \\
\text { MEcc- }\end{array}$ & 3504.682646 & 428.3711 & 0 & 0 \\
\hline 1 & $\begin{array}{l}\text { polymer } \\
\text { DISPV- }\end{array}$ & $\begin{array}{c}3847.571353 \\
-\end{array}$ & 429.1625 & $\begin{array}{c}0.317179979 \\
-\end{array}$ & 1.211387 \\
\hline 2 & $\begin{array}{l}\text { polymer } \\
\text { Mor17m- }\end{array}$ & $\begin{array}{c}15.25520772 \\
-\end{array}$ & 1.028103 & $\begin{array}{c}0.665154575 \\
-\end{array}$ & 1.530487 \\
\hline 3 & $\begin{array}{l}\text { polymer } \\
\text { MDDD- }\end{array}$ & $\begin{array}{c}302.3633458 \\
-\end{array}$ & 37.40017 & $\begin{array}{c}0.267519932 \\
-\end{array}$ & 1.129238 \\
\hline 4 & $\begin{array}{l}\text { solvent } \\
\text { pilD- }\end{array}$ & 31.81118727 & 2.123853 & 1.096516046 & 2.498295 \\
\hline 5 & $\begin{array}{l}\text { solvent } \\
\text { X3V- }\end{array}$ & 18.36408016 & 1.027251 & 1.620295289 & 3.093179 \\
\hline 6 & $\begin{array}{l}\text { solvent } \\
\text { MATS2m- }\end{array}$ & 25.16525596 & 6.381213 & 0.201238909 & 1.741354 \\
\hline 7 & $\begin{array}{l}\text { solvent } \\
\text { Mor25p- }\end{array}$ & $\begin{array}{c}39.50802607 \\
-\end{array}$ & 7.341934 & $\begin{array}{c}0.229734722 \\
-\end{array}$ & 1.456877 \\
\hline 8 & solvent & 393.3137093 & 51.41867 & 0.347300595 & 1.549369 \\
\hline
\end{tabular}

Tab. 4. The eight molecular descriptors entered into the best obtained multi-linear equation.

\begin{tabular}{|c|c|c|c|}
\hline ID & $\begin{array}{l}\text { Molecular } \\
\text { Descriptor }\end{array}$ & Type & Definition \\
\hline & MEcc- & Geometrical & \\
\hline 1 & $\begin{array}{l}\text { polymer } \\
\text { DISPV- }\end{array}$ & Geometrical & $\begin{array}{l}\text { Molecular eccentricity } \\
\text { d COMMA@ value/weighted by atomic }\end{array}$ \\
\hline 2 & polymer & Descriptors & van der Waals volumes \\
\hline 3 & $\begin{array}{l}\text { Mor17m- } \\
\text { polymer }\end{array}$ & $\begin{array}{l}\text { 3D-MoRSE } \\
\text { descriptors }\end{array}$ & $\begin{array}{l}\text { 3D-MoRSE-signal 17/weighted by } \\
\text { atomic masses }\end{array}$ \\
\hline 4 & $\begin{array}{l}\text { MDDD- } \\
\text { solvent }\end{array}$ & $\begin{array}{l}\text { Topological } \\
\text { Descriptors }\end{array}$ & Mean distance degree deviation \\
\hline & & Walk and path & \\
\hline 5 & pilD-solvent & $\begin{array}{l}\text { counts } \\
\text { Connecivity }\end{array}$ & Conventional bond-order ID number \\
\hline 6 & X3v-solvent & indices & Valence connectivity index chi-3 \\
\hline 7 & $\begin{array}{l}\text { MATS2m- } \\
\text { solvent }\end{array}$ & $\begin{array}{l}\text { 2D } \\
\text { autoco }\end{array}$ & $\begin{array}{l}\text { Moran autocorrelation -lag2/weighted by } \\
\text { atomic masses }\end{array}$ \\
\hline 8 & $\begin{array}{l}\text { Mor25p- } \\
\text { solvent }\end{array}$ & $\begin{array}{l}\text { 3D-MoRSE } \\
\text { descriptors }\end{array}$ & $\begin{array}{l}\text { 3D-MoRSE-signal 25/weighted by } \\
\text { atomic polarizabilities }\end{array}$ \\
\hline
\end{tabular}


Tab. 5. The statistical parameters of fitting and QSPR analysis parameters.

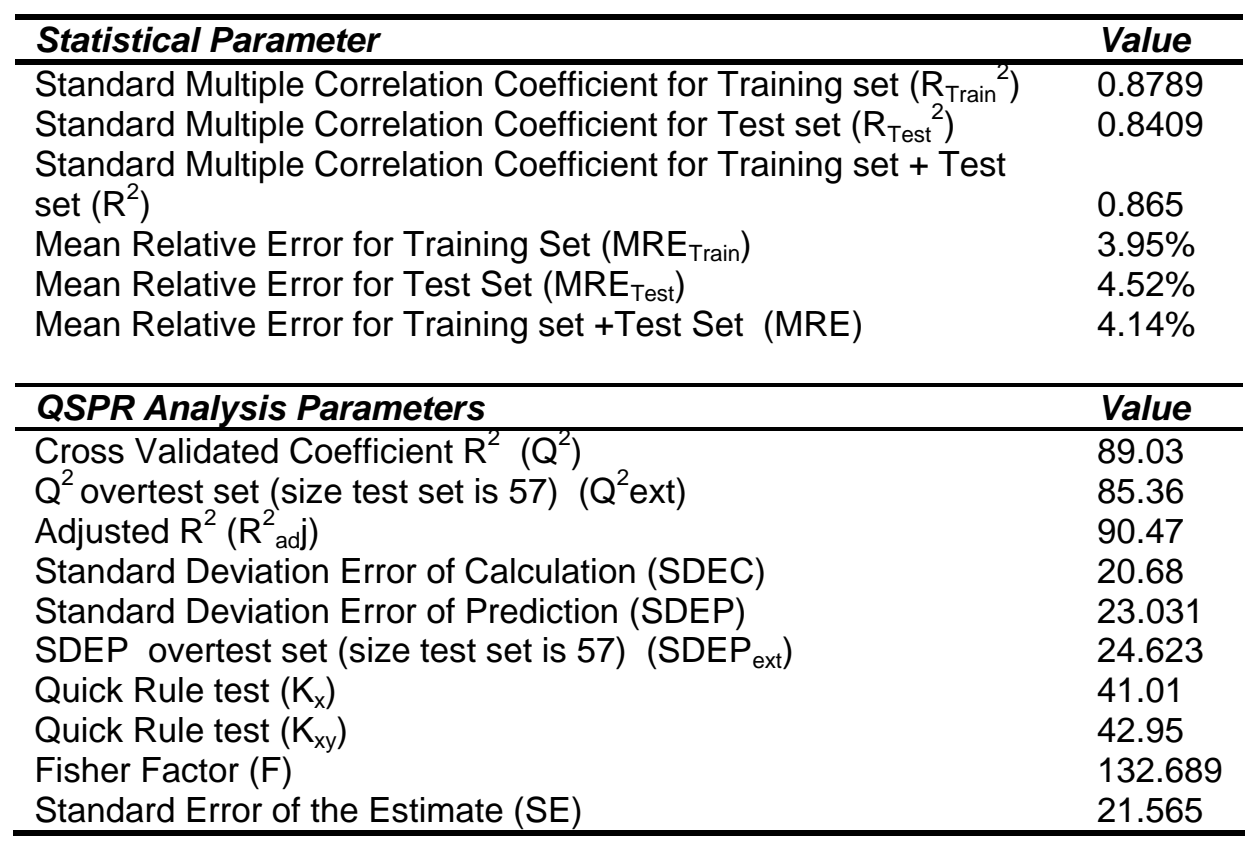

The behavior of the obtained multivariate linear model (eight-parameter linear equation) presented in Tab. 3, is shown in Fig. 2.

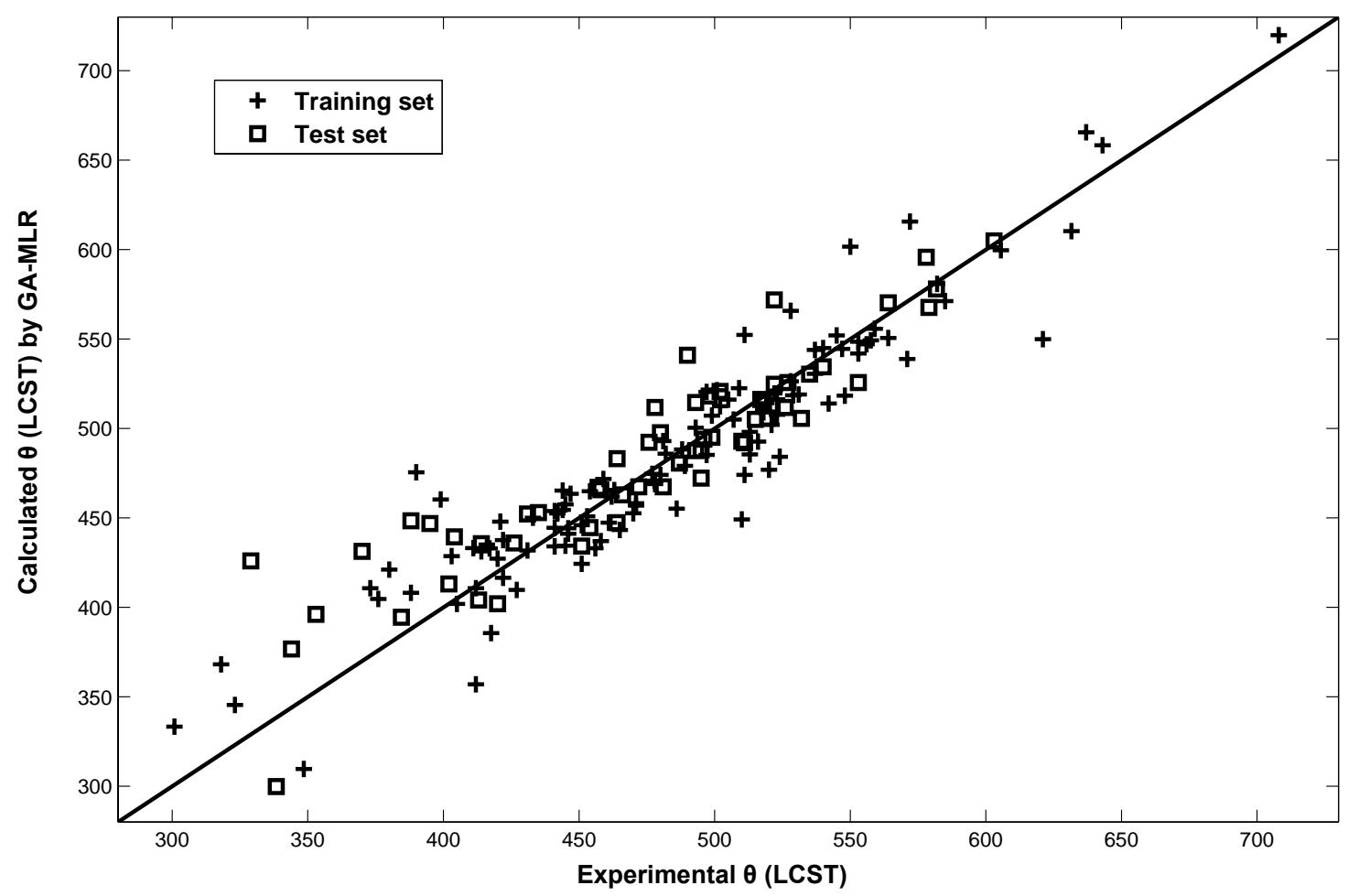

Fig. 2. Comparison between the best multi-linear results obtained by GA-MLR and the experimental data. 
In this study the best multivariate linear model which is obtained, is an eightparameter equation. This equation and its statistical parameters are presented in the Tab. 3. The physical meanings of molecular descriptors which entered in the best multi-linear equation are presented in Tab. 4. The values of these eight molecular descriptors for all 169 polymer solutions are presented as supplementary materials. Also the statistical parameters of fitting this correlation are presented in Tab. 5.

The MRE of the model over all experimental data is $4.14 \%$. This value is quite equal to the MRE of the previous works, but the number of parameters of presented model is less than previous models. This obtained result is the first part of calculations.

\section{Neural Network Calculations}

The three-layer feed forward neural networks with the sigmoidal (hyperbolic tangent) transfer function have been the standard techniques used in QSPR modeling [24]. In order to consider the nonlinear behavior of different molecular descriptors which were entered into the best multivariate linear model, obtained in previous section, threelayer feed forward neural networks (FFNN) were used.

This part of calculations was done in Neural Network Toolbox, available in MATLAB software (Mathworks Inc. Software).

In this section, the structure of the three-layer FFNN which was used to study is explained step by step during solving the problem.

The first work is dividing the main dataset into two datasets, first for training network and second for testing it. Neural networks are good at fitting functions and there is a proof that a simple neural network can fit any dataset very well; as a result for checking the prediction power of the neural network, usage of test set is needed. Test set is only used for checking the produced neural network and is not used to train it. In this work 57 polymer solutions of 169 polymer solutions were randomly allocated to test set and all remaining 112 polymer solutions were allocated to training set.

The schematic of the three-layer FFNN which was used in this work are shown in Fig. 2.

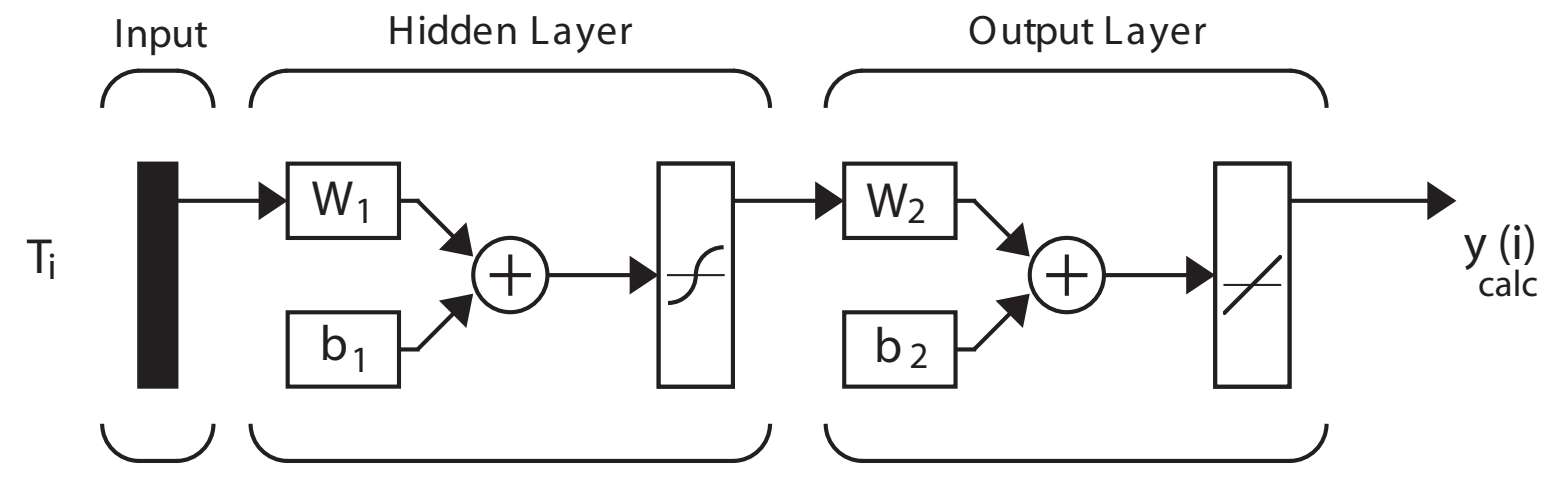

Fig. 3. The schematic structure of three layer feed forward neural network used in this study.

We can simplify the results of the three-layer FFNN in the form of Eq. (1). 
$y_{\text {calc }}(i)=\left(W_{2} \times\left(\tanh \left(\left(W_{1} \times T_{i}\right)+b_{1}\right)\right)\right)+b_{2}$

In this equation, $T$ is the input matrix and is a nparam $\times$ nds matrix where nparam is the number of molecular descriptors (it is equal to 8 in this work) and $n d s$ is the number of polymer solutions available in the training set (it is equal to 112 in this problem). $T_{i}$ is the ith-column of the matrix $T . W_{1}$ is the weight matrix of the second layer in our FFNN and is a $n \times n d s$ matrix which $n$ is the number of neurons in the hidden layer. $b_{1}$ is the bias matrix of second layer and is a $n \times 1$ matrix. $W_{2}$ is the weight matrix of output layer (third layer) and is a $n \times 1$ matrix. $b_{2}$ is the bias of output layer (third layer) which is a scalar quantity. $y_{\text {calc }}(i)$ is he ith output of this network which must be compared with ith physical property.

Usually, all inputs and outputs of FFNN are normalized between -1 and +1 , for decreasing the accounting error. This work was performed in this section, by means of the minimum and maximum values of any descriptors as well as output values.

The values of $W_{1}, W_{2}, b_{1}$, and $b_{2}$ are obtained by minimization of an objective function which is commonly the mean squared error between the outputs of neural network and the target values. This minimization is usually done by LevenbergMarquart algorithm. This algorithm is rapid and accurate in process of training neural networks [24].

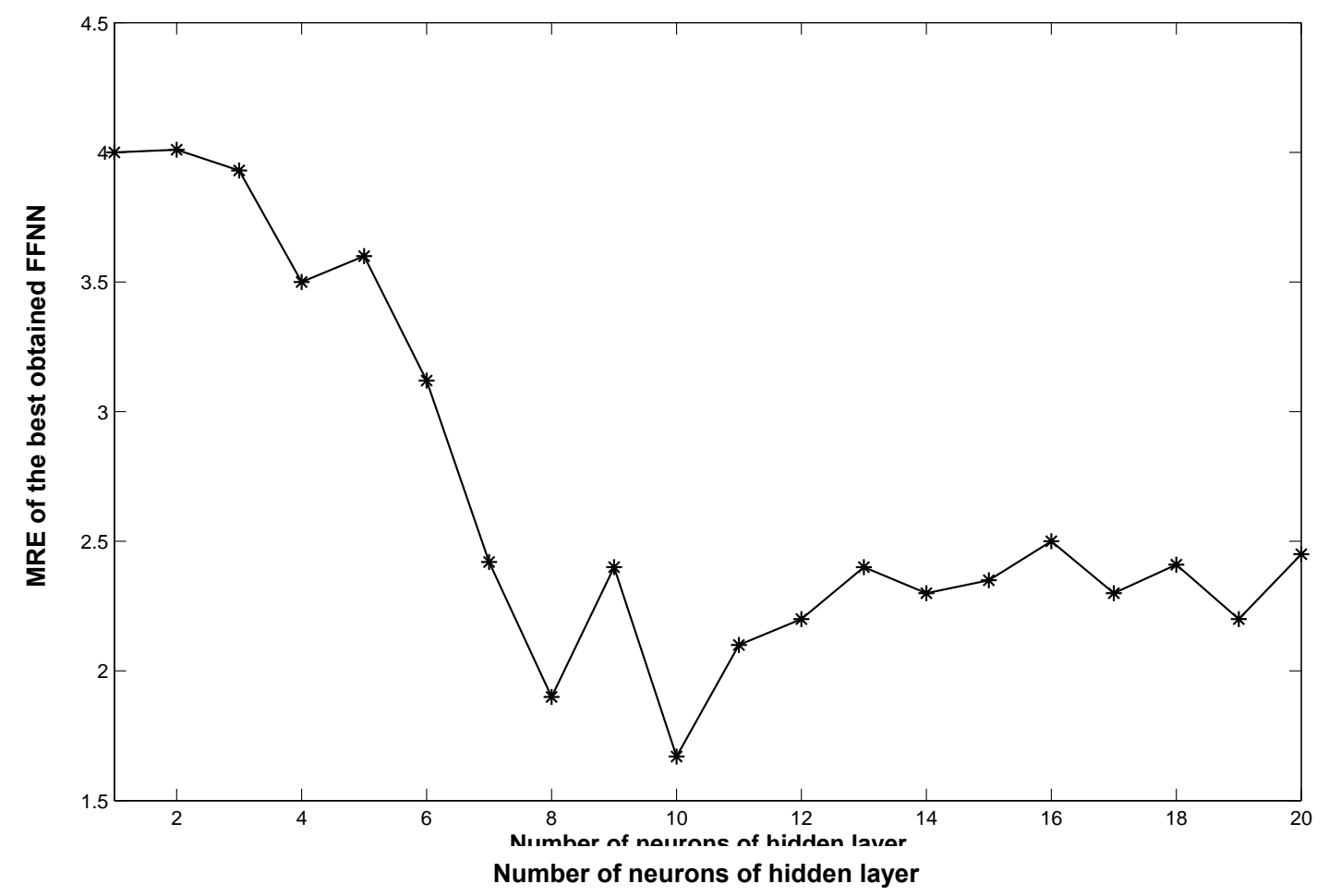

Fig. 4. Effect of number of neurons of hidden layer on the MRE of the best obtained FFNN.

In most cases first the number of neurons of hidden layer $(n)$ is fixed, then it is tried to produce a neural network which can predict the target values with the best accuracy. Then this work is repeated till the best neural network is obtained. In many 
cases especially in three-layer FFNNs, it is better the number of neurons of hidden layer is optimized, as a complementary work.

In this work, a three-layer FFNN was generated to predict $\theta(L C S T)$ of polymer solutions, then the number of neurons of hidden layer of this three-layer FFNN was post-optimized for obtaining the best number of neurons of hidden layer. As can be seen in Fig. 3, the best number of neurons of hidden layer which is obtained is equal to 10. It should be noted that all FFNNs with structure 8-1-1 to 8-20-1 were checked to obtain the best FFNNs. The MRE of these FFNNs are shown in the Fig. 3.

The best three-layer FFNN which was obtained for prediction $\theta(L C S T)$ of polymer solutions has the structures 8-10-1 and shows $R^{2}=0.9736$ and MRE $1.67 \%$ over all experimental data set. The values of $W_{1}, W_{2}, b_{1}$, and $b_{2}$ for this FFNN are presented in Tab. 6. Also the predicted values of $\theta(L C S T)$ in comparison to experimental data are presented in Fig. 1.

Tab. 6. Weight and bias matrices of the best obtained three layer feed forward neural network.

\begin{tabular}{|c|c|c|c|c|c|c|c|c|c|}
\hline$W_{1}$ & & & & & & & & & $b_{1}$ \\
\hline 0.3113 & 0.8756 & -0.708 & 0.798 & 0.1394 & 0.0439 & 1.5048 & -0.696 & & 2.1366 \\
\hline 0.5547 & 1.0573 & 0.7505 & 0.0824 & 0.5761 & 0.9691 & 0.5074 & 0.3306 & & 1.4044 \\
\hline 0.8431 & 1.0351 & 0.7658 & -0.322 & 0.0074 & 0.0077 & 0.3078 & 0.6849 & & 1.0165 \\
\hline-1.541 & 1.2856 & 0.1388 & 0.6983 & 0.2058 & 0.4043 & 0.2871 & 0.8182 & & 0.7645 \\
\hline 0.2015 & 0.1992 & 0.6909 & 0.1303 & 1.1088 & 1.1097 & 0.4414 & 0.2572 & & 0.6198 \\
\hline 0.0455 & 0.8549 & 0.0712 & 0.6278 & -0.982 & 0.1141 & 0.1184 & 0.1027 & & 0.4453 \\
\hline 0.5488 & 0.8558 & 1.2487 & 0.0169 & 0.1544 & 0.6864 & 0.7333 & -0.685 & & 0.3654 \\
\hline 0.964 & 1.1757 & 0.4145 & 0.5717 & 0.2343 & 0.2172 & 0.2889 & -0.943 & & 0.8297 \\
\hline 0.4037 & $19222^{-}$ & $1.7512^{-}$ & $0.4542^{-}$ & 0.9982 & 0.2037 & 0.8422 & -0.163 & & 0.3371 \\
\hline 0.3544 & 0.3598 & 0.4066 & 0.3509 & -1.173 & 1.2595 & 0.3784 & 0.7987 & & -1.55 \\
\hline \multicolumn{10}{|l|}{$W_{2}$} \\
\hline 0.7419 & 0.5111 & 0.2933 & 0.5444 & 0.4939 & 1.3342 & 0.0265 & 0.4012 & 0.542 & 0.7205 \\
\hline \multicolumn{10}{|l|}{$b_{2}$} \\
\hline 0.1496 & & & & & & & & & \\
\hline
\end{tabular}

The statistical parameters of the obtained FFNN for prediction of $\theta(L C S T)$ are presented in Tab. 5.

The obtained MRE by this FFNN over all experimental data is $1.67 \%$. This value, in comparison to previous works, shows considerable modification. 


\section{References}

[1] Elias, H.G. Theta Solvents, VII-291- VII-326, in Polymer Handbook, Brandrup, J.; Immergut H.; Grulke E.A., edtors, Wiley, New York, 1999.

[2] Kavanagh, C.A.; Rochev Y.A.; Gallangher, W.M.; Dawson, K.A; Keenan, A.K. Pharmacol. Ther. 2004, 102, 1.

[3] Kopecek, J. Eur. Pharm. Sci. 2003, 20, 1.

[4] Todeschini R.; Consonni, V.; in: Manhold R.; Kubinyi H.; Temmerman, H. (Series editors), Handbook of molecular descriptors, Weinheim, Wiley-VCH, 2000.

[5] Melagraki, G.; Afantitis, A.; Sarimves, H.; Koutenis, P.A.; Markopoulos, J.; IglessiMarkopoulou, O. J. Mol. Model. 2007, 13, 55.

[6] Chang, B.H.; Bae, C.Y. Polymer 1998, 39, 6449.

[7] Pappa, G.D.; Voutsas, E.C.; Tassios, D.P. Ind. Eng. Chem. Res. 2001, 40, 4654.

[8] Bogdanic, G.; Vidal, J. Fluid Phase Equilib. 2000, 173, 241.

[9] Imre, A.R.; Bae, Y.C.; Chang, B.H.; Kraska, T. Ind. Eng. Chem. Res. 2004, 43, 237.

[10] Vetere, A. Ind. Eng. Chem. Res. 1998, 48, 2864.

[11] Vetere, A. Ind. Eng. Chem. Res. 1998, 37, 4463.

[12] Wang, F.; Saeki, S.; Yamaguchi, T. Polymer 1999, 40, 2779.

[13] Kayi, H.; Tuncel, S.A.; Elkamel, A.; Alper, E., J. Mol. Model. 2005, 11, 55.

[14] Liu, H.; Zhong, C.; Eur. Polym. J. 2005, 41, 139.

[15] Xu, J.; Liu, L.; Xu, W.; Zhao, S.;, Zuo, D. J. Mol. Graph. Model. (2007), doi: 10.1016/j.jmgm.2007.01.004.

[16] Gharagheizi, F. Comput. Mater. Sci. 2007, 40, 159.

[17] Liu., H.; Zhong, C. Ind. Eng. Chem. Res. 2005, 44, 634.

[18] HyperChem Release 7.5 for Windows, Molecular Modeling System, Hypercube, Inc., 2002.

[19] Talete srl, Dragon for windows (Software for molecular Descriptor Calculations). Version 5.4 - 2006 - http://www/talete.mi.it/

[20] Leardi, R.; Boggia, R.; Terrile, M. J. Chemometr. 1992, 6, 267.

[21] Vatani, A.; Mehrpooya, M.; Gharagheizi, F. Int. J. Mol. Sci. 2007, 8, 407.

[22] Gharagheizi, F.; Mehrpooya, M. Energ. Convers. Manage. 2007, 48, 2453.

[23] Gharagheizi, F. QSAR Comb. Sci. 2007, doi: 10.1002/qsar.200630159.

[24] Taskinen, J.; Yliruusi, J. Adv. Drug Deliv. Rev. 2003, 55, 1163. 
Supplementary Materials

\begin{tabular}{|c|c|c|c|c|c|c|c|c|c|c|}
\hline \multirow[b]{2}{*}{ ID } & \multirow[b]{2}{*}{ Polymer } & \multirow[b]{2}{*}{ Solvent } & \multicolumn{8}{|c|}{ Molecular Descriptors } \\
\hline & & & $\begin{array}{c}\text { Mecc- } \\
\text { polymer }\end{array}$ & $\begin{array}{c}\text { DISPV- } \\
\text { polymer }\end{array}$ & $\begin{array}{l}\text { Mor17m- } \\
\text { polymer }\end{array}$ & $\begin{array}{l}\text { MDDD- } \\
\text { solvent }\end{array}$ & $\begin{array}{c}\text { pilD- } \\
\text { solvent }\end{array}$ & $\begin{array}{c}X 3 v- \\
\text { solvent }\end{array}$ & $\begin{array}{l}\text { MATS2m- } \\
\text { solvent }\end{array}$ & $\begin{array}{c}\text { Mor25p- } \\
\text { solvent }\end{array}$ \\
\hline 1 & P4MP1 & n-Butane & 0.998 & 0.626 & -0.138 & 1 & 7.178 & 0.5 & 1 & 0.1 \\
\hline 2 & P4MP1 & n-Pentane & 0.998 & 0.626 & -0.138 & 1.6 & 9.787 & 0.707 & 1 & 0.092 \\
\hline 3 & P4MP1 & n-Heptane & 0.998 & 0.626 & -0.138 & 2.857 & 15.525 & 1.207 & 1 & 0.094 \\
\hline 4 & P4MP1 & n-Octane & 0.998 & 0.626 & -0.138 & 4 & 18.605 & 1.457 & 1 & 0.1 \\
\hline 5 & P4MP1 & 2-Methyl butane & 0.998 & 0.626 & -0.138 & 1.36 & 9.317 & 0.816 & 1 & 0.116 \\
\hline 6 & P4MP1 & 2,2-Dimethyl butane & 0.998 & 0.626 & -0.138 & 1.556 & 11.257 & 1.061 & 1 & 0.123 \\
\hline 7 & P4MP1 & 2,2-Dimethyl pentane & 0.998 & 0.626 & -0.138 & 2.122 & 14.139 & 1 & 1 & 0.113 \\
\hline 8 & P4MP1 & $2,2,3$-Trimethyl butane & 0.998 & 0.626 & -0.138 & 2 & 13.194 & 1.732 & 1 & 0.089 \\
\hline 9 & P4MP1 & Cyclopentane & 0.998 & 0.626 & -0.138 & 0 & 12.167 & 1.25 & 1 & 0.16 \\
\hline 10 & P4MP1 & n-Hexane & 0.998 & 0.626 & -0.138 & 2.222 & 12.579 & 0.957 & 1 & 0.093 \\
\hline 11 & P4MP1 & n-Nonane & 0.998 & 0.626 & -0.138 & 5.185 & 21.802 & 1.707 & 1 & 0.102 \\
\hline 12 & P4MP1 & 2,4-Dimethyl pentane & 0.998 & 0.626 & -0.138 & 2.612 & 14.244 & 0.943 & 1 & 0.108 \\
\hline 13 & P4MP1 & 3-Ethyl pentane & 0.998 & 0.626 & -0.138 & 2.816 & 14.224 & 1.732 & 1 & 0.069 \\
\hline 14 & PB1 & n-Pentane & 0.999 & 0 & -0.099 & 1.6 & 9.787 & 0.707 & 1 & 0.092 \\
\hline 15 & PB1 & n-Heptane & 0.999 & 0 & -0.099 & 2.857 & 15.525 & 1.207 & 1 & 0.094 \\
\hline 16 & PB1 & n-Octane & 0.999 & 0 & -0.099 & 4 & 18.605 & 1.457 & 1 & 0.1 \\
\hline 17 & PB1 & 2-Methyl butane & 0.999 & 0 & -0.099 & 1.36 & 9.317 & 0.816 & 1 & 0.116 \\
\hline 18 & PB1 & 2,2-Dimethyl butane & 0.999 & 0 & -0.099 & 1.556 & 11.257 & 1.061 & 1 & 0.123 \\
\hline 19 & PB1 & $2,2,3$-Trimethyl butane & 0.999 & 0 & -0.099 & 2 & 13.194 & 1.732 & 1 & 0.089 \\
\hline 20 & PB1 & 2,5-Dimethyl hexane & 0.999 & 0 & -0.099 & 3.5 & 17.287 & 1.321 & 1 & 0.144 \\
\hline 21 & PB1 & 3-Ethyl pentane & 0.999 & 0 & -0.099 & 2.816 & 14.224 & 1.732 & 1 & 0.069 \\
\hline 22 & PB1 & 3,4-Dimethyl hexane & 0.999 & 0 & -0.099 & 3 & 16.776 & 2.259 & 1 & 0.066 \\
\hline 23 & PB1 & n-Hexane & 0.999 & 0 & -0.099 & 2.222 & 12.579 & 0.957 & 1 & 0.093 \\
\hline 24 & PB1 & n-Nonane & 0.999 & 0 & -0.099 & 5.185 & 21.802 & 1.707 & 1 & 0.102 \\
\hline 25 & PB1 & 2,4-Dimethyl pentane & 0.999 & 0 & -0.099 & 2.612 & 14.244 & 0.943 & 1 & 0.108 \\
\hline 26 & PB1 & 2,3-Dimethyl pentane & 0.999 & 0 & -0.099 & 2.408 & 14.07 & 1.782 & 1 & 0.074 \\
\hline 27 & PB1 & Cyclopentane & 0.999 & 0 & -0.099 & 0 & 12.167 & 1.25 & 1 & 0.16 \\
\hline 28 & PBD & n-Hexane & 0.999 & 2.972 & 0.002 & 2.222 & 12.579 & 0.957 & 1 & 0.093 \\
\hline 29 & PBD & $2,2,3$-Trimethyl butane & 0.999 & 2.972 & 0.002 & 2 & 13.194 & 1.732 & 1 & 0.089 \\
\hline 30 & PBD & n-Octane & 0.999 & 2.972 & 0.002 & 4 & 18.605 & 1.457 & 1 & 0.1 \\
\hline 31 & PBD & Ethyl propyl ketone & 0.999 & 2.972 & 0.002 & 2.612 & 15.659 & 0.923 & -0.222 & 0.171 \\
\hline 32 & PBD & Diethyl ketone & 0.999 & 2.972 & 0.002 & 2 & 12.664 & 0.789 & -0.28 & 0.006 \\
\hline 33 & PBD & 2-Methyl hexane & 0.999 & 2.972 & 0.002 & 2.735 & 14.986 & 1.135 & 1 & 0.114 \\
\hline
\end{tabular}


2,2,4-Trimethyl

$\begin{array}{lllll}34 & \text { PBD } & \text { pentane } & 0.999 & 2.972 \\ 35 & \text { PBD } & \text { Propylene oxide } & 0.999 & 2.972 \\ 36 & \text { PDMS } & \text { n-Pentane } & 0.989 & 1.194 \\ 37 & \text { PDMS } & \text { n-Heptane } & 0.989 & 1.194 \\ 38 & \text { PDMS } & \text { n-Octane } & 0.989 & 1.194 \\ 39 & \text { PDMS } & \text { n-Dodecane } & 0.989 & 1.194 \\ 40 & \text { PDMS } & \text { n-Cetane } & 0.989 & 1.194 \\ 41 & \text { PDMS } & \text { n-Hexane } & 0.989 & 1.194 \\ 42 & \text { PDMS } & \text { n-Decane } & 0.989 & 1.194 \\ 43 & \text { PE } & \text { n-Hexane } & 0.989 & 0 \\ 44 & \text { PE } & \text { n-Heptane } & 0.989 & 0 \\ 45 & \text { PE } & \text { n-Nonane } & 0.989 & 0 \\ 46 & \text { PE } & \text { n-Decane } & 0.989 & 0 \\ 47 & \text { PE } & \text { n-Dodecane } & 0.989 & 0 \\ 48 & \text { PE } & \text { n-Tridecane } & 0.989 & 0 \\ 49 & \text { PE } & \text { 2,2-Dimethyl pentane } & 0.989 & 0 \\ 50 & \text { PE } & \text { 2,2,3-Trimethyl butane } & 0.989 & 0 \\ 51 & \text { PE } & \text { 2,3-Dimethyl pentane } & 0.989 & 0 \\ 52 & \text { PE } & \text { 3-Ethyl pentane } & 0.989 & 0 \\ & & \text { 2,2,4,4-Tetramethyl } & & \\ 53 & \text { PE } & \text { pentane } & 0.989 & 0 \\ 54 & \text { PE } & \text { 2,3,4-Trimethyl hexane } & 0.989 & 0 \\ 55 & \text { PE } & \text { Methyl cyclopentane } & 0.989 & 0 \\ 56 & \text { PE } & \text { Methyl cyclohexane } & 0.989 & 0 \\ 57 & \text { PE } & \text { 1-Octanol } & 0.989 & 0 \\ 58 & \text { PE } & \text { n-Pentyl acetate } & 0.989 & 0 \\ 59 & \text { PE } & \text { n-Pentane } & 0.989 & 0 \\ 60 & \text { PE } & \text { n-Octane } & 0.989 & 0 \\ 61 & \text { PE } & \text { n-Undecane } & 0.989 & 0 \\ 62 & \text { PE } & \text { 2,4-Dimethyl pentane } & 0.989 & 0 \\ & & \text { 2,2,4-Trimethyl } & & \\ 63 & \text { PE } & \text { pentane } & 0.989 & 0 \\ 64 & \text { PE } & \text { 3,4-Dimethyl hexane } & 0.989 & 0 \\ 65 & \text { PE } & \text { Cyclopentane } & 0.989 & 0 \\ 66 & \text { PE } & \text { Cyclohexane } & 0.989 & 0 \\ 67 & \text { PE } & \text { n-Butyl acetate } & 0.989 & 0 \\ 68 & \text { PIB } & \text { Cyclopentane } & 0.985 & 0.804 \\ 69 & \text { PIB } & \text { Cyclohexane } & 0.804\end{array}$

\begin{tabular}{l}
0.002 \\
0.002 \\
-0.312 \\
-0.312 \\
-0.312 \\
-0.312 \\
-0.312 \\
-0.312 \\
-0.312 \\
-0.048 \\
-0.048 \\
-0.048 \\
-0.048 \\
-0.048 \\
-0.048 \\
-0.048 \\
-0.048 \\
-0.048 \\
-0.048 \\
\\
\hline-0.048 \\
-0.048 \\
-0.048 \\
-0.048 \\
-0.048 \\
-0.048 \\
-0.048 \\
-0.048 \\
-0.048 \\
-0.048 \\
\\
-0.048 \\
-0.048 \\
-0.048 \\
-0.048 \\
-0.048 \\
-0.076 \\
-0.076
\end{tabular}

$\begin{array}{ccc}2.875 & 16.215 & 1.021 \\ 0.5 & 8.5 & 0.333 \\ 1.6 & 9.787 & 0.707 \\ 2.857 & 15.525 & 1.207 \\ 4 & 18.605 & 1.457 \\ 9 & 31.987 & 2.457 \\ 16.25 & 46.672 & 3.457 \\ 2.222 & 12.579 & 0.957 \\ 6.4 & 25.104 & 1.957 \\ 2.222 & 12.579 & 0.957 \\ 2.857 & 15.525 & 1.207 \\ 5.185 & 21.802 & 1.707 \\ 6.4 & 25.104 & 1.957 \\ 9 & 31.987 & 2.457 \\ 10.769 & 35.552 & 2.707 \\ 2.122 & 14.139 & 1 \\ 2 & 13.194 & 1.732 \\ 2.408 & 14.07 & 1.782 \\ 2.816 & 14.224 & 1.732 \\ & & \\ 3.259 & 18.085 & 1.061 \\ 3.506 & 19.038 & 2.593 \\ 1 & 15.283 & 1.644 \\ 1.143 & 18.967 & 1.894 \\ 5.185 & 21.802 & 1.512 \\ 4.494 & 22.043 & 1.212 \\ 1.6 & 9.787 & 0.707 \\ 4 & 18.605 & 1.457 \\ 7.636 & 28.502 & 2.207 \\ 2.612 & 14.244 & 0.943 \\ 2.875 & 16.215 & 1.021 \\ 3 & 16.776 & 2.259 \\ 0 & 12.167 & 1.25 \\ 0 & 15.73 & 1.5 \\ 3.5 & 18.791 & 0.962 \\ 0 & 12.167 & 1.25 \\ 0 & 15.73 & 1.5\end{array}$

1
-0.333
1
1
1
1
1
1
1
1
1
1
1
1
1
1
1
1
1
1
1
1
1
1
-0.036
0.366
1
1
1
1
1
1
1
1
1
1
1
1
1
1
1
1
1
1
1
1
1
1
1
1
1
1
1
1
1

0.122

0.053

0.092

0.094

0.1

0.106

0.108

0.093

0.096

0.093

0.094

0.102

0.096

0.106

0.109

0.113

0.089

0.074

0.069

0.121

0.029

0.182

0.244

0.079

0.104

0.092

0.1

0.097

0.108

0.122

0.066

0.16

0.219

0.081

0.16

0.219 


\begin{tabular}{|c|c|c|c|c|c|c|c|c|c|c|}
\hline 70 & PIB & Cycloheptane & 0.985 & 0.804 & -0.076 & 0 & 19.477 & 1.75 & 1 & 0.136 \\
\hline 71 & PIB & Cyclooctane & 0.985 & 0.804 & -0.076 & 0 & 23.381 & 2 & 1 & 0.202 \\
\hline 72 & PIB & n-Heptane & 0.985 & 0.804 & -0.076 & 2.857 & 15.525 & 1.207 & 1 & 0.094 \\
\hline 73 & PIB & n-Octane & 0.985 & 0.804 & -0.076 & 4 & 18.605 & 1.457 & 1 & 0.1 \\
\hline 74 & PIB & n-Dodecane & 0.985 & 0.804 & -0.076 & 9 & 31.987 & 2.457 & 1 & 0.106 \\
\hline 75 & PIB & 2-Methyl butane & 0.985 & 0.804 & -0.076 & 1.36 & 9.317 & 0.816 & 1 & 0.116 \\
\hline 76 & PIB & 2-Methyl pentane & 0.985 & 0.804 & -0.076 & 2 & 12.068 & 0.866 & 1 & 0.108 \\
\hline 77 & PIB & 3-Methyl pentane & 0.985 & 0.804 & -0.076 & 2 & 11.886 & 1.394 & 1 & 0.102 \\
\hline 78 & PIB & 3-Methyl hexane & 0.985 & 0.804 & -0.076 & 2.612 & 14.763 & 1.478 & 1 & 0.101 \\
\hline 79 & PIB & 3-Ethyl pentane & 0.985 & 0.804 & -0.076 & 2.816 & 14.224 & 1.732 & 1 & 0.069 \\
\hline 80 & PIB & 2,3-Dimethyl pentane & 0.985 & 0.804 & -0.076 & 2.408 & 14.07 & 1.782 & 1 & 0.074 \\
\hline 81 & PIB & 2,4-Dimethyl pentane & 0.985 & 0.804 & -0.076 & 2.612 & 14.244 & 0.943 & 1 & 0.108 \\
\hline 82 & PIB & $2,2,3$-Trimethyl butane & 0.985 & 0.804 & -0.076 & 2 & 13.194 & 1.732 & 1 & 0.089 \\
\hline 83 & PIB & Ethyl cyclopentane & 0.985 & 0.804 & -0.076 & 1.755 & 18.59 & 2.052 & 1 & 0.236 \\
\hline 84 & PIB & 3-Methyl heptane & 0.985 & 0.804 & -0.076 & 3.5 & 17.794 & 1.747 & 1 & 0.108 \\
\hline 85 & PIB & 2,2-Dimethyl hexane & 0.985 & 0.804 & -0.076 & 3.063 & 17.17 & 1.28 & 1 & 0.126 \\
\hline 86 & PIB & 2,5-Dimethyl hexane & 0.985 & 0.804 & -0.076 & 3.5 & 17.287 & 1.321 & 1 & 0.144 \\
\hline 87 & PIB & 3.4-Dimethyl hexane & 0.985 & 0.804 & -0.076 & 3 & 16.776 & 2.259 & 1 & 0.066 \\
\hline 88 & PIB & n-Propyl cyclopentane & 0.985 & 0.804 & -0.076 & 2.75 & 22.037 & 2.172 & 1 & 0.193 \\
\hline 89 & PIB & n-Pentane & 0.985 & 0.804 & -0.076 & 1.6 & 9.787 & 0.707 & 1 & 0.092 \\
\hline 90 & PIB & n-Hexane & 0.985 & 0.804 & -0.076 & 2.222 & 12.579 & 0.957 & 1 & 0.093 \\
\hline 91 & PIB & Methyl cyclopentane & 0.985 & 0.804 & -0.076 & 1.143 & 18.967 & 1.894 & 1 & 0.244 \\
\hline 92 & PIB & 2-Methyl hexane & 0.985 & 0.804 & -0.076 & 2.735 & 14.986 & 1.135 & 1 & 0.114 \\
\hline 93 & PIB & 2,2-Dimethyl pentane & 0.985 & 0.804 & -0.076 & 2.122 & 14.139 & 1 & 1 & 0.113 \\
\hline 94 & PIB & 3,3-Dimethyl pentane & 0.985 & 0.804 & -0.076 & 2.204 & 13.782 & 1.914 & 1 & 0.102 \\
\hline 95 & PIB & Methyl cyclohexane & 0.985 & 0.804 & -0.076 & 1.143 & 18.967 & 1.894 & 1 & 0.244 \\
\hline 96 & PIB & 2-Methyl heptane & 0.985 & 0.804 & -0.076 & 3.75 & 18.045 & 1.385 & 1 & 0.119 \\
\hline 97 & PIB & $\begin{array}{l}\text { 2,4-Dimethyl hexane } \\
\text { 2,2,4-Trimethyl }\end{array}$ & 0.985 & 0.804 & -0.076 & 3.25 & 17.113 & 1.571 & 1 & 0.107 \\
\hline 98 & PIB & pentane & 0.985 & 0.804 & -0.076 & 2.875 & 16.215 & 1.021 & 1 & 0.122 \\
\hline 99 & PIB & n-Decane & 0.985 & 0.804 & -0.076 & 6.4 & 25.104 & 1.957 & 1 & 0.096 \\
\hline 100 & PMMA & Methyl acetate & 0.997 & 1.818 & -0.052 & 1.36 & 10.124 & 0.287 & 0.042 & 0.06 \\
\hline 101 & PMMA & Ethyl acetate & 0.997 & 1.818 & -0.052 & 2 & 12.664 & 0.516 & 0.2 & 0.083 \\
\hline 102 & PMMA & Ethyl propyl ketone & 0.997 & 1.818 & -0.052 & 2.612 & 15.659 & 0.923 & -0.222 & 0.028 \\
\hline 103 & PMMA & Diethyl ketone & 0.997 & 1.818 & -0.052 & 2 & 12.664 & 0.789 & -0.28 & 0.006 \\
\hline 104 & PMMA & Butyl chloride & 0.997 & 1.818 & -0.052 & 1.6 & 9.787 & 0.754 & -0.167 & 0.058 \\
\hline 105 & PMMA & Dipropyl ketone & 0.997 & 1.818 & -0.052 & 3.313 & 18.568 & 1.058 & -0.184 & 0.051 \\
\hline 106 & PMMA & Methyl ethyl ketone & 0.997 & 1.818 & -0.052 & 1.36 & 10.124 & 0.498 & -0.375 & 0.097 \\
\hline 107 & PMMA & Tetrahydrofuran & 0.997 & 1.818 & -0.052 & 0 & 12.167 & 0.827 & -0.25 & 0.153 \\
\hline
\end{tabular}




\begin{tabular}{|c|c|c|c|c|c|c|c|c|c|c|}
\hline 108 & PMS & Cyclopentane & 0.999 & 8.008 & -0.083 & 0 & 12.167 & 1.25 & 1 & 0.16 \\
\hline 109 & PMS & Cyclohexane & 0.999 & 8.008 & -0.083 & 0 & 15.73 & 1.5 & 1 & 0.219 \\
\hline 110 & PMS & Butyl chloride & 0.999 & 8.008 & -0.083 & 1.6 & 9.787 & 0.754 & -0.167 & 0.058 \\
\hline 111 & PMS & n-Butyl acetate & 0.999 & 8.008 & -0.083 & 3.5 & 18.791 & 0.962 & 0.333 & 0.081 \\
\hline 112 & PMS & n-Hexyl acetate & 0.999 & 8.008 & -0.083 & 5.6 & 25.4 & 1.462 & 0.389 & 0.097 \\
\hline 113 & PMS & Methyl cyclohexane & 0.999 & 8.008 & -0.083 & 1.143 & 18.967 & 1.894 & 1 & 0.244 \\
\hline 114 & PMS & n-Pentyl acetate & 0.999 & 8.008 & -0.083 & 4.494 & 22.043 & 1.212 & 0.366 & 0.087 \\
\hline 115 & PP & n-Pentane & 0.996 & 0.658 & -0.067 & 1.6 & 9.787 & 0.707 & 1 & 0.092 \\
\hline 116 & $\mathrm{PP}$ & n-Hexane & 0.996 & 0.658 & -0.067 & 2.222 & 12.579 & 0.957 & 1 & 0.093 \\
\hline 117 & $\mathrm{PP}$ & n-Octane & 0.996 & 0.658 & -0.067 & 4 & 18.605 & 1.457 & 1 & 0.1 \\
\hline 118 & PP & n-Nonane & 0.996 & 0.658 & -0.067 & 5.185 & 21.802 & 1.707 & 1 & 0.102 \\
\hline 119 & $\mathrm{PP}$ & 2,2-Dimethyl butane & 0.996 & 0.658 & -0.067 & 1.556 & 11.257 & 1.061 & 1 & 0.123 \\
\hline 120 & PP & 2,3-Dimethyl butane & 0.996 & 0.658 & -0.067 & 1.778 & 11.347 & 1.333 & 1 & 0.09 \\
\hline 121 & $\mathrm{PP}$ & 2,2-Dimethyl pentane & 0.996 & 0.658 & -0.067 & 2.122 & 14.139 & 1 & 1 & 0.113 \\
\hline 122 & $\mathrm{PP}$ & 2,2,3-Trimethyl butane & 0.996 & 0.658 & -0.067 & 2 & 13.194 & 1.732 & 1 & 0.089 \\
\hline 123 & $\mathrm{PP}$ & 2,3-Dimethyl pentane & 0.996 & 0.658 & -0.067 & 2.408 & 14.07 & 1.782 & 1 & 0.074 \\
\hline 124 & PP & $\begin{array}{l}\text { 3-Ethyl pentane } \\
2,2,4,4 \text {-Tetramethyl }\end{array}$ & 0.996 & 0.658 & -0.067 & 2.816 & 14.224 & 1.732 & 1 & 0.069 \\
\hline 125 & $\mathrm{PP}$ & pentane & 0.996 & 0.658 & -0.067 & 3.259 & 18.085 & 1.061 & 1 & 0.121 \\
\hline 126 & $\mathrm{PP}$ & 2,3,4-Trimethyl hexane & 0.996 & 0.658 & -0.067 & 3.506 & 19.038 & 2.593 & 1 & 0.029 \\
\hline 127 & $\mathrm{PP}$ & Methyl cyclopentane & 0.996 & 0.658 & -0.067 & 1 & 15.283 & 1.644 & 1 & 0.181 \\
\hline 128 & $\mathrm{PP}$ & Methyl cyclohexane & 0.996 & 0.658 & -0.067 & 1.143 & 18.967 & 1.894 & 1 & 0.244 \\
\hline 129 & PP & Diethyl ether & 0.996 & 0.658 & -0.067 & 1.6 & 9.787 & 0.408 & -0.583 & 0.166 \\
\hline 130 & $\mathrm{PP}$ & n-Heptane & 0.996 & 0.658 & -0.067 & 2.857 & 15.525 & 1.207 & 1 & 0.094 \\
\hline 131 & PP & 2-Methyl butane & 0.996 & 0.658 & -0.067 & 1.36 & 9.317 & 0.816 & 1 & 0.116 \\
\hline 132 & PP & $\begin{array}{l}\text { 2,4-Dimethyl pentane } \\
\text { 2,2,4-Trimethyl }\end{array}$ & 0.996 & 0.658 & -0.067 & 2.612 & 14.244 & 0.943 & 1 & 0.108 \\
\hline 133 & $\mathrm{PP}$ & pentane & 0.996 & 0.658 & -0.067 & 2.875 & 16.215 & 1.021 & 1 & 0.122 \\
\hline 134 & PP & 3,4-Dimethyl hexane & 0.996 & 0.658 & -0.067 & 3 & 16.776 & 2.259 & 1 & 0.066 \\
\hline 135 & $\mathrm{PP}$ & Cyclopentane & 0.996 & 0.658 & -0.067 & 0 & 12.167 & 1.25 & 1 & 0.16 \\
\hline 136 & $\mathrm{PP}$ & Cyclohexane & 0.996 & 0.658 & -0.067 & 0 & 15.73 & 1.5 & 1 & 0.219 \\
\hline 137 & PP1 & n-Pentane & 0.999 & 0.532 & -0.138 & 1.6 & 9.787 & 0.707 & 1 & 0.092 \\
\hline 138 & PP1 & n-Hexane & 0.999 & 0.532 & -0.138 & 2.222 & 12.579 & 0.957 & 1 & 0.093 \\
\hline 139 & PP1 & n-Octane & 0.999 & 0.532 & -0.138 & 4 & 18.605 & 1.457 & 1 & 0.1 \\
\hline 140 & PP1 & 2-Methyl butane & 0.999 & 0.532 & -0.138 & 1.36 & 9.317 & 0.816 & 1 & 0.116 \\
\hline 141 & PP1 & 2,4-Dimethyl pentane & 0.999 & 0.532 & -0.138 & 2.612 & 14.244 & 0.943 & 1 & 0.108 \\
\hline 142 & PP1 & 2,2-Dimethyl pentane & 0.999 & 0.532 & -0.138 & 2.122 & 14.139 & 1 & 1 & 0.113 \\
\hline 143 & PP1 & 2,3-Dimethyl pentane & 0.999 & 0.532 & -0.138 & 2.408 & 14.07 & 1.782 & 1 & 0.074 \\
\hline 144 & PP1 & 3-Methyl heptane & 0.999 & 0.532 & -0.138 & 3.5 & 17.794 & 1.747 & 1 & 0.108 \\
\hline
\end{tabular}




\begin{tabular}{|c|c|c|c|c|c|c|c|c|c|c|}
\hline 145 & PP1 & n-Heptane & 0.999 & 0.532 & -0.138 & 2.857 & 15.525 & 1.207 & 1 & 0.094 \\
\hline 146 & PP1 & $\begin{array}{l}\text { 2,2-Dimethyl butane } \\
\text { 2,2,4-Trimethyl }\end{array}$ & 0.999 & 0.532 & -0.138 & 1.556 & 11.257 & 1.061 & 1 & 0.123 \\
\hline 147 & PP1 & pentane & 0.999 & 0.532 & -0.138 & 2.875 & 16.215 & 1.021 & 1 & 0.122 \\
\hline 148 & PP1 & Cyclopentane & 0.999 & 0.532 & -0.138 & 0 & 12.167 & 1.25 & 1 & 0.16 \\
\hline 149 & PPCS & Isobutyl acetate & 1 & 13.623 & 0.033 & 3.25 & 17.97 & 0.803 & 0.333 & 0.122 \\
\hline 150 & PPCS & Ethyl carbitol & 1 & 13.623 & 0.033 & 7.636 & 28.502 & 1.152 & -0.542 & 0.315 \\
\hline 151 & PPCS & n-Butyl carbitol & 1 & 13.623 & 0.033 & 10.769 & 35.552 & 1.59 & -0.409 & 0.371 \\
\hline 152 & PPCS & tert-Butyl acetate & 1 & 13.623 & 0.033 & 2.563 & 16.713 & 0.798 & 0.333 & 0.156 \\
\hline 153 & PS & Cyclopentane & 1 & 6.513 & -0.066 & 0 & 12.167 & 1.25 & 1 & 0.16 \\
\hline 154 & PS & Cyclohexane & 1 & 6.513 & -0.066 & 0 & 15.73 & 1.5 & 1 & 0.219 \\
\hline 155 & PS & Methyl cyclopentane & 1 & 6.513 & -0.066 & 1 & 15.283 & 1.644 & 1 & 0.181 \\
\hline 156 & PS & Methyl cyclohexane & 1 & 6.513 & -0.066 & 1.143 & 18.967 & 1.894 & 1 & 0.244 \\
\hline 157 & PS & Benzene & 1 & 6.513 & -0.066 & 0 & 21.32 & 0.667 & 1 & 0.34 \\
\hline 158 & PS & Toluene & 1 & 6.513 & -0.066 & 1.143 & 25.971 & 0.94 & 1 & 0.301 \\
\hline 159 & PS & Ethyl acetate & 1 & 6.513 & -0.066 & 2 & 12.664 & 0.516 & 0.2 & 0.083 \\
\hline 160 & PS & n-Propyl acetate & 1 & 6.513 & -0.066 & 2.612 & 15.659 & 0.682 & 0.283 & 0.137 \\
\hline 161 & PS & i-Propyl acetate & 1 & 6.513 & -0.066 & 2.408 & 14.783 & 0.673 & 0.3 & 0.124 \\
\hline 162 & PS & Ethyl n-butyrate & 1 & 6.513 & -0.066 & 3.313 & 18.568 & 0.759 & 0.143 & 0.152 \\
\hline 163 & PS & Isobutyl acetate & 1 & 6.513 & -0.066 & 3.25 & 17.97 & 0.803 & 0.333 & 0.122 \\
\hline 164 & PS & sec-Butyl acetate & 1 & 6.513 & -0.066 & 3 & 17.565 & 1.216 & 0.333 & 0.131 \\
\hline 165 & PS & tert-Butyl acetate & 1 & 6.513 & -0.066 & 2.563 & 16.713 & 0.798 & 0.333 & 0.184 \\
\hline 166 & PS & Diethyl malonate & 1 & 6.513 & -0.066 & 6.05 & 28.004 & 0.899 & -0.071 & 0.09 \\
\hline 167 & PS & i-Amyl acetate & 1 & 6.513 & -0.066 & 3.802 & 20.868 & 1.44 & 0.357 & 0.085 \\
\hline 168 & PS & Methyl acetate & 1 & 6.513 & -0.066 & 1.36 & 10.124 & 0.287 & 0.042 & 0.06 \\
\hline 169 & PS & Methyl ethyl ketone & 1 & 6.513 & -0.066 & 1.36 & 10.124 & 0.498 & -0.375 & 0.068 \\
\hline
\end{tabular}

